\title{
Archéopages
}

Archéopages

Archéologie et société

45 | 2017

De la terre au pot

\section{Photogrammétrie, lasergrammétrie, tomodensitométrie}

Des outils numériques pour la caractérisation des chaînes opératoires céramiques

Photogrammetry, 3D laser scanning, computed tomography. Digital tools for the characterization of ceramic operational chains

Fotogrametría, lasergrametría, tomodensitometría. Herramientas digitales para caracterizar cadenas operatorias cerámicas

Théophane Nicolas, Geneviève Treyvaud, Quentin Favrel, Ronan Gaugne et Valérie Gouranton

\section{OpenEdition}

\section{Journals}

Édition électronique

URL : https://journals.openedition.org/archeopages/3483

DOI : $10.4000 /$ archeopages.3483

ISSN : 2269-9872

Éditeur

INRAP - Institut national de recherches archéologiques préventives

Édition imprimée

Date de publication : 1 mars 2018

ISSN : $1622-8545$

\section{Référence électronique}

Théophane Nicolas, Geneviève Treyvaud, Quentin Favrel, Ronan Gaugne et Valérie Gouranton,

"Photogrammétrie, lasergrammétrie, tomodensitométrie », Archéopages [En ligne], 45 | 2017, mis en ligne le 01 janvier 2020, consulté le 04 juin 2021. URL : http://journals.openedition.org/archeopages/ 3483 ; DOI : https://doi.org/10.4000/archeopages.3483 


\section{Photogrammétrie, lasergrammétrie, tomodensitométrie}

\section{Des outils numériques pour la caractérisation des chaînes opératoires céramiques}

Théophane Nicolas Irrap, UMR 8215 «Trajectoires"

Geneviève Treyvaud INss-Eau Terre Environnement

Quentin Favrel Université Paris IPantheon-Sorbonne, LMR 8225 « Trajectoires"

Ronan Gaugne Universite Rernes, UMR 607 «IRISA"

Valérie Gouranton INSA Rennes, UMR 607\& «IRISA "

\section{6}

1. Tomodensitométrie: reconstructions 3D

surfacique d'un gobelet

campaniforme sur

les axes $X$ et $Y$.
L'utilisation de moyens d'investigation non invasive à haute complexité technologique pour l'analyse du mobilier archéologique est actuellement limitée, réservée le plus souvent à des découvertes exceptionnelles. Ici, au contraire, sont exposés les méthodes et les résultats de l'utilisation de quelques-uns de ces moyens pour l'étude du mobilier fréquent, courant, banal que sont les céramiques protohistoriques.
Ces recherches récentes ont été menées dans le cadre d'un projet ANR [encadré 1] visant à utiliser des technologies d'imagerie avancées (tomodensitométrie...) et de restitutions ${ }_{3} \mathrm{D}$ interactives, physiques et virtuelles (réalité virtuelle, augmentée, mixte ; impressions $3 \mathrm{D}$ et interactions tangibles) dans le but d'affiner la lecture d'objets complexes, composites et/ou altérés.

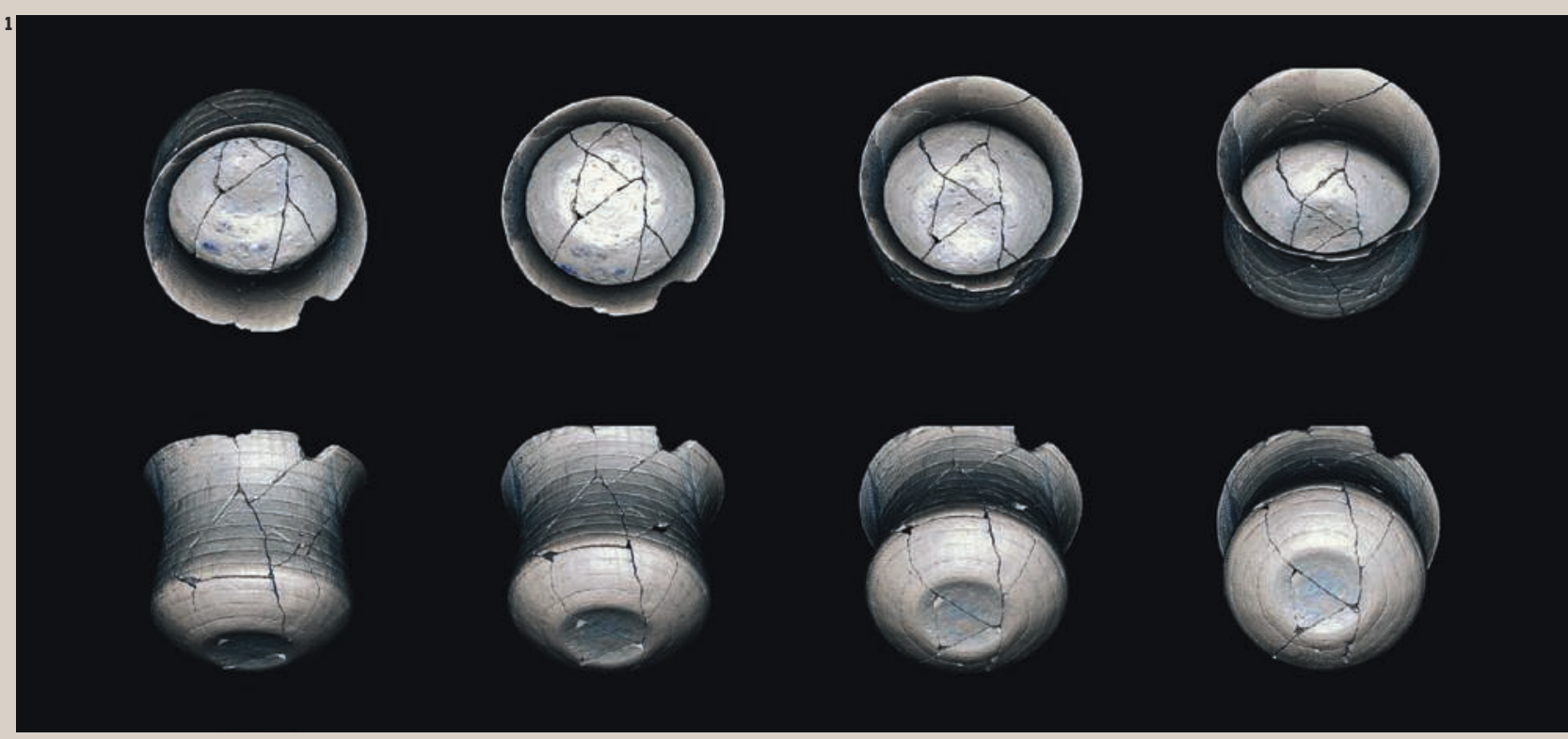




\section{Trois méthodes}

Si l'on s'intéresse aux publications récentes sur l'usage de la tomodensitométrie [encadré 2] pour l'analyse de mobilier archéologique, on remarque qu'elles portent essentiellement sur des momies et des squelettes humains ou animaux (HouleWierzbicki et al., 2015 ; Xiujie et al., 2009 ; Ikram et al., 2015) ou des objets exceptionnels (Mödlinger, 2008 ; Hassmann et al., 2012) et que peu de travaux examinent les autres types de mobiliers (Huisman et al., 2014 ; Lee et al., 2008). Ces dernières années, l'usage de cette technologie s'est néanmoins développé dans le domaine de l'étude des incinérations (Anderson, Fell, 1995 ; Minozzi et al., 2010 ; Harving et al., 2012 ; Harving, Lynnerup, 2013 ; Le Puil-Texier et al., 2015) et lors de découvertes d'ensembles particulièrement complexes nécessitant des prélèvements en «bloc » souvent en lien avec des opérations de conservation ou de fouilles complexes (Stelzner et al., 2010 ; Nicolas et al., 2014 ; Nicolas et al., 2015, Re et al., 2015), ou bien grâce à l'intérêt porté à la tomodensitométrie par des géologues et sédimentologues (Hu et al., 2006 ; Ketcham, Iturrino, 2005). Certaines de ces publications ont facilité le développement de l'application en archéologie de cette technique (Kak, Slaney, 1999; Ketcham, Carlson, 2001) notamment pour la caractérisation des matériaux archéologiques et des modes de fabrication d'objets, dans un premier temps métalliques (Mödlinger, 2008 ; Treyvaud, 2010, 2015 ; Treyvaud et al., 2013) et plus récemment céramiques (Bouzakis et al., 2011 ; Kahl et al., 2012).

Par ailleurs, depuis ces dernières années, la numérisation tridimensionnelle avec la démocratisation d'outils (suite logicielle, scanner $3 \mathrm{D}$...) suscite un intérêt grandissant de la part de la communauté archéologique. Ce type d'acquisition (tout particulièrement la photogrammétrie et la lasergrammétrie) offre une alternative attrayante pour la documentation, l'analyse et le partage des données du mobilier archéologique par rapport aux méthodes traditionnelles en deux dimensions (photographie, dessin...) [ill. 1 et 2a]. L'état de l'art montre que la numérisation $3 \mathrm{D}$ est utilisée principalement pour l'établissement de données précises de manière non invasive et sans avoir à manipuler les objets - obtention de coupes virtuelles ou calcul de caractères difficiles à mesurer par les moyens traditionnels (volume, surface ou symétrie) (Forte, Kurrillo, 2010). Les auteurs s'accordent à dire qu'il s'agit d'une méthode efficace pour créer des modèles numériques et physiques, imprimables (Barreau et al., 2014) ou manipulables (Arbace et al., 2013). En outre, la numérisation 3D crée des modèles pérennes facilement accessibles. Si ces méthodes permettent de s'affranchir des échelles par rapport aux méthodes traditionnelles, elles restent toutefois le plus souvent cantonnées à la surface des objets et nécessitent, à ce jour, un matériel onéreux et des ressources informatiques importantes (cartes graphiques...). Les deux techniques, photogrammétrie [ill. 2b] et lasergrammétrie [ill. 2c], sont opérantes à des degrés divers dans le cadre d'une analyse céramologique. Grâce à la photogrammétrie, on obtient un modèle texturé et de manipulation aisée. L'inconvénient est que le modèle n'est pas toujours particulièrement pertinent à cause d'un manque de précision du relevé de surface. Ce défaut peut être corrigé par la capacité des logiciels de traitement à s'affranchir des textures ; après optimisation, on accède à une meilleure lecture de certaines macrotraces, ou à des analyses quantifiables de variations de surface. On dispose alors de paramètres quantitatifs décrivant la

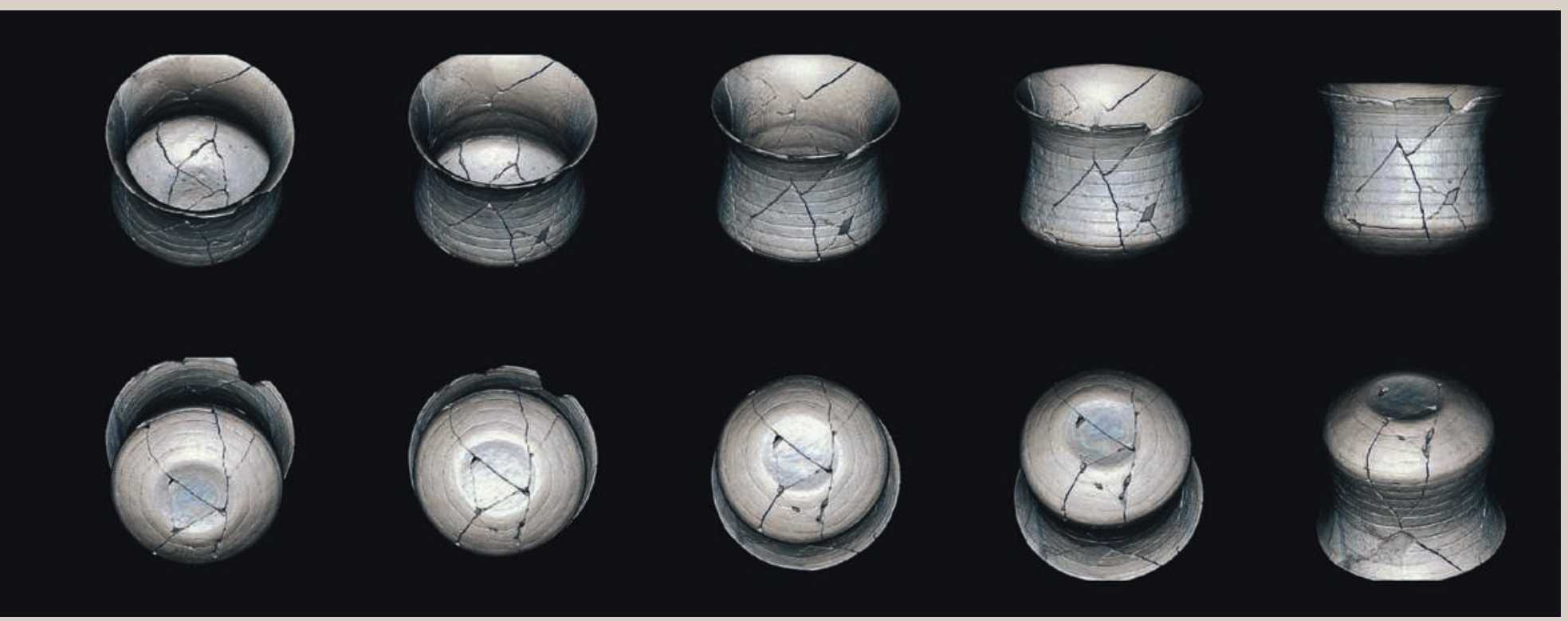




\section{Le projet Introspect}

La représentation virtuelle des mobiliers archéologiques et des sédiments qui les composent, leur reconstruction dans l'espace et une copie obtenue par impression 3D, voire leur manipulation en réalité virtuelle, ne sont qu'une part des technologies accessibles aux chercheurs en archéologie. Ces dernières années se sont beaucoup développées des méthodes de numérisation qui apportent des solutions non destructives d'étude, de conservation et de présentation du patrimoine archéologique. Cependant, ces techniques sont limitées à la surface visible des objets, des monuments ou des sites. Les informations structurelles et contextuelles du mobilier archéologique sont des aspects primordiaux. C'est pour dépasser les limites de ces moyens technologiques que plusieurs chercheurs ont formé le projet INTROSPECT (Introspection du mobilier archéologique à l'ère du numérique). Il repose sur une collaboration entre chercheurs en informatique et archéologie en France (Insa de Rennes,
Université de Rennes 1, UMR 6074 « IRISA ", Inrap, et UMRR 6566 " CReAAF ») et au Québec (Université Laval, INRS). Il est soutenu financièrement par

l'Agence nationale de la recherche française (ANR-16-FRQC-004) et par le Fonds de recherche du Québec - société et culture (FQSC) pour une durée de trois ans (Biron et al., 2015 ; Nicolas et al., 2014, 2015). Ce projet vise à développer de nouveaux usages et outils qui facilitent l'accès des archéologues à de nouvelles connaissances : méthodes d'introspection numérique interactive combinant la tomodensitométrie avec des technologies de visualisation 3D, telles la réalité virtuelle, les interactions tangibles et l'impression 3D. La multidisciplinarité apporte un défi méthodologique important, d'autant plus que l'approche retenue fait appel à la mutualisation de méthodes utilisées en ingénierie, réalité virtuelle, archéologie et muséologie.

www.introspect.info
La tomodensitométrie théorique et pratique La tomodensitométrie - en anglais " computed tomography " (CT) - est le nom scientifique de la scanographie par rayons $\mathrm{X}$, technique d'imagerie très utilisée dans l'imagerie médicale [ill. 1]. Son but est de permettre l'acquisition axiale d'un objet et de le soustraire aux effets de superposition obtenus en radiographie classique. Les rayons $X$ sont produits par un générateur $\mathrm{X}$. Le tube générant les rayons $\mathrm{X}$ comme le détecteur situé en face du tube émetteur de rayons $X$ tournent autour de l'objet à analyser. Ces éléments sont contenus dans l'anneau entourant la table sur laquelle est placé l'objet. On obtient ainsi des images en coupe d'un objet à trois dimensions et le cumul de ces images permet la reconstruction 3D dudit objet [ill. 2].

La tomodensitométrie réalise une cartographie d'un artefact et un enregistrement systématique en valeur HU (cf. infra) des densités des matériaux le composant ainsi que sa lecture en trois dimensions. Cette analyse de la matière au cœur des objets, non destructive, fournit des paramètres qualitatifs et quantitatifs sur les matériaux utilisés afin de définir et de comprendre les structures internes de l'objet, son mode de fabrication et son état de conservation. Il est envisagé de faire de même pour les sédiments archéologiques. Des méthodes numériques sont utilisées pour reconstruire en 3D la matrice de densité relative d'un objet. Pour ce faire, les mesures de projections par des détecteurs sont d'abord réalisées en éclairant à l'aide d'une source à rayons X l'objet sur 360 degrés dans son axe transversal. Les mesures des détecteurs sont appelées des projections et elles sont proportionnelles au ratio de l'intensité émise du faisceau $\left(I_{0}\right)$ et l'intensité mesurée par les détecteurs (I), selon la loi de Beer-Lambert. Ce ratio dépend du coefficient d'atténuation du faisceau $(\boldsymbol{\mu})$ déterminé, entre autres, par le numéro atomique de la matière. La tomodensitométrie cherche donc à déterminer la matrice 3D des coefficients d'atténuation de l'objet. Pour le CT scan médical, les valeurs d'atténuation générées sont des valeurs relatives par rapport à celle de l'eau ( $\left.\mu_{\text {eau }}\right)$ et sont appelées des valeurs HU. Pour reconstruire cette matrice d'atténuation 3D, on utilise l'algorithme analytique de la rétroprojection filtrée. Celui-ci se base sur le théorème de Radon qui stipule que seule la mesure de l'ensemble des projections de l'objet, appelé sinogramme, permet d'en reconstituer l'image (C. Bourgault-Brunelle, INRS-FTE 2017 ). Depuis les années 2000 , cette méthode est appliquée dans le domaine de la géologie et de la recherche sur les matériaux industriels. Bien qu'utilisée déjà en archéologie, pour l'étude des corps humains ou d'animaux momifiés, et en paléontologie, pour l'étude des fossiles, elle est peu employée pour l'étude technologique et de caractérisation du mobilier archéologique (Ketcham, Carlson, 2001). Le scanner est composé d'un ensemble sourcedétecteurs en rotation autour d'un objet archéologique déposé sur une table d'examen radiotransparente. La puissance émise, la collimation -l'orientation précise du détecteur -, la dimension et le nombre de points focaux sont des éléments paramétrables du tube à rayons $\mathbf{X}$. Il est donc possible d'adapter les paramètres de la machine en fonction de l'objet à étudier et selon les questions posées. L'excellente résolution spatiale de ces appareils, de l'ordre maximal de $0,1 \mathrm{~mm}$, permet de voir des détails très subtils : les structures des matériaux, les stratifications fines, les assemblages, les inclusions, la consolidation et la déformation d'un corps. L'appareil peut analyser rapidement des objets d'un diamètre maximal de $50 \mathrm{~cm}$ et de $250 \mathrm{~cm}$ de longueur. 


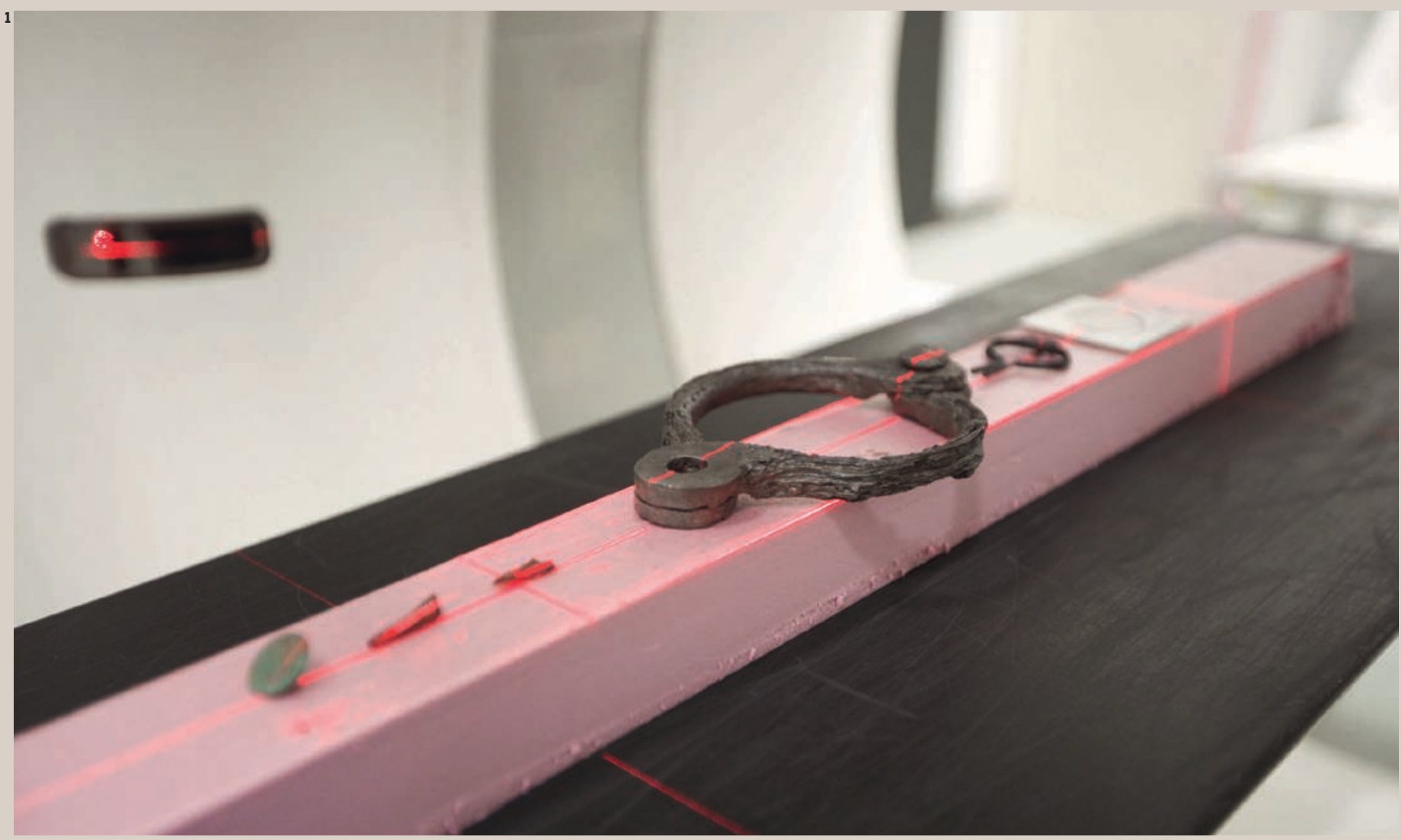

1. Scanner, et mobilier en cours d'acquisition. 2. Schéma de fonctionnement d'un scanner: a: anneau;

b: tube émetteur de rayons $X$ c: détecteur; d: rayon $X$;

e: images en coupe, ce sont ces coupes qui permettront une reconstruction en trois dimensions.
La tomodensitométrie donnant ainsi à l'archéologue la possibilité de voir le mobilier archéologique en trois dimensions et d'en comprendre la structure interne apparaît tout indiquée pour l'étude de la technologie. Les données obtenues lors de l'acquisition sont traitées de deux façons. La reconstruction à l'aide de logiciels d'imagerie donne accès à des images segmentaires, ciblées ou complètes de l'artefact. Par exemple, on peut choisir d'étudier un vase céramique dans son ensemble en 3D pour voir le dessin général d'un décor, de segmenter le vase par coupes de moins d'un millimètre pour identifier le mode de fabrication, identifier des inclusions comme des dégraissants ou encore caractériser des fissures et des réparations. Il est aussi possible de compléter les analyses visuelles en utilisant des logiciels de calcul (comme Matlab) pour déterminer le pourcentage de porosité d'une matière, le pourcentage de dégraissant, les variations de la densité, le volume d'une cavité, etc. Cependant, comme tout moyen technologique, la tomodensitométrie a quelques limites d'usage. Les principales sont les dimensions de l'anneau et de la table, la quantification de la densité réelle des matériaux à partir des densités tomographiques HU et enfin, la gestion des effets de « beam hardening " particulièrement présents lors du traitement des matériaux métalliques. Ces effets sont dus à des différences de densité très élevées au sein de la structure traversée, au voisinage de matière de forte densité ou de produit de contraste en concentration élevée, et entraînent un durcissement non uniforme du faisceau. On distingue alors sur l'image une ombre dans et immédiatement à côté d'une structure à haut contraste. Il est actuellement impossible d'identifier d'une façon théorique et avec exactitude la densité réelle des éléments étudiés.
Nous avons donc cherché à gérer ces restrictions. Afin de caractériser le ou les matériaux qui composent l'objet archéologique, nous avons passé dans l'appareil, à une très haute résolution, des échantillons de divers matériaux dont nous avions toutes les caractéristiques physiques et chimiques. Les résultats obtenus nous ont permis de bâtir une échelle de comparaison des densités HU et réelles ainsi qu'un répertoire des caractéristiques physicochimique des matériaux archéologiques (Boespflug et al., 1994). On a pu ainsi, par comparaison, identifier la densité réelle d'un matériau grâce à cette échelle. Concernant les effets de " beam hardening", il est possible de les minimiser en isolant l'artefact dans un contenant de sable ou encore de les contrôler ou d'en diminuer l'impact par des améliorations des algorithmes de reconstruction utilisés lors du traitement des données brutes.

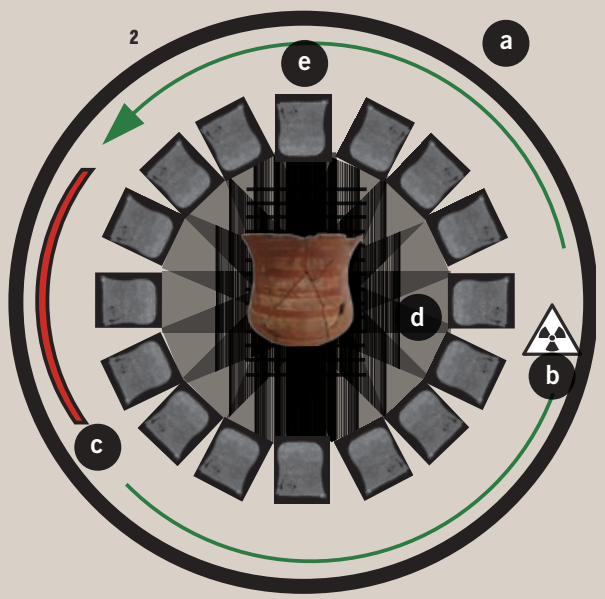


2. Gobelet campaniforme.

a. Vue photographique.

b. Vues en photogrammétrie.

c. Tomodensitométrie:

vues VRT (Volume Rendering

Technique).
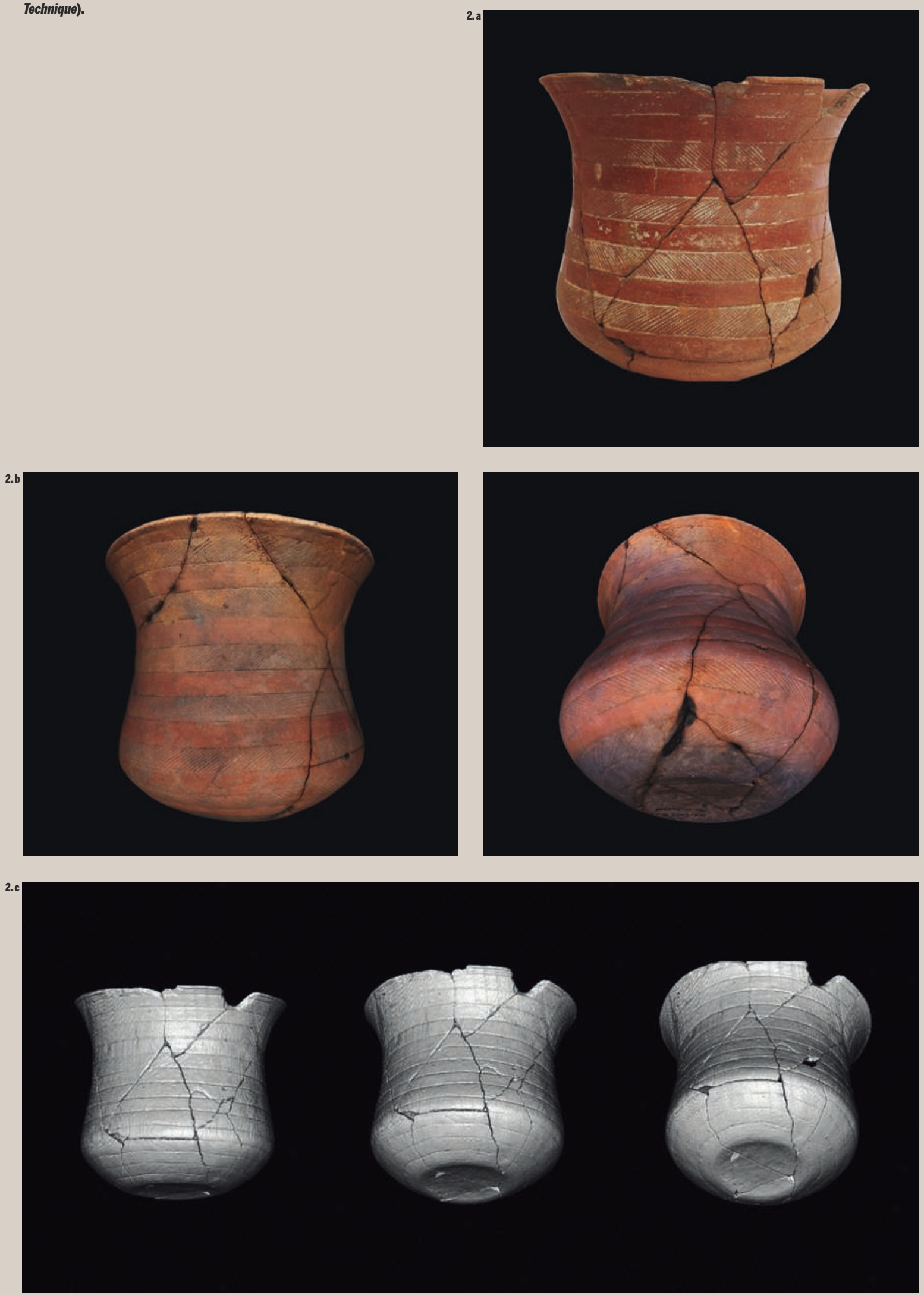
morphologie des récipients (profil et volume), et, avec des résolutions d'acquisition pouvant atteindre $0,1 \mathrm{~mm}$ avec un scanner $3 \mathrm{D}$, il devient envisageable de caractériser plus finement les stigmates de finition, les variations de surface ou les registres décoratifs de la céramique. La résolution fine est susceptible de révéler la géométrie des empreintes d'un décor et de déterminer la morphologie des outils utilisés. La mise en évidence de la variabilité des outils utilisés et de leurs empreintes est un moyen de restituer la gestuelle du potier. Toutefois, ces deux techniques ont des limites : d'abord, la morphologie complexe de certains objets, qui empêche, par exemple, l'étude de l'intérieur des récipients à profils rentrants ou à reliefs prononcés ; ensuite l'oblitération de certaines opérations de finition - de façonnage, par exemple - par d'autres traitements postérieurs, ce qui réduit l'analyse à quelques portions de la chaîne opératoire, en général aux dernières étapes de celle-ci.

Le scanner (ou tomodensitomètre) est un appareil de radiologie qui utilise des rayons $\mathrm{X}$ afin d'explorer différents organes du corps humain. L'exploration s'effectue par deux « passages » ou acquisitions, un mode radio et une acquisition spiralée. Le mode radio, encore appelé topogramme, aboutit à une visualisation de l'objet de la même façon qu'une radiographie « classique ». Il a pour but de permettre de positionner les coupes à réaliser, des « tranches » de l'objet considéré, images planaires (en 2D) successives de celui-ci [ill. 3a]. Elles sont reconstruites à partir d'une acquisition hélicoïdale (ou spiralée) des profils d'atténuation de l'objet (appelé sinogramme). On peut ainsi déterminer les premières informations sur l'objet et les densités (échelle d'Hounsfield-en niveau de gris). Ces images ont une épaisseur de o,75 $\mathrm{mm}$, reconstruites tous les $0,2 \mathrm{~mm}$ dans l'axe de découpe $(Z)$, soit une résolution de o,2 $\mathrm{mm}$ dans cette axe identique à la résolution dans le plan de découpe $(\mathrm{XY})$. On parle alors d'isotropisme de l'information permettant une reconstruction multi-planaire (MPR) de qualité identique aux images acquises. Les images subissent également un traitement lorsqu'on les reconstruit par le biais de différents filtres, dits de convolution :

- Filtre dur (passe-haut [B7of]) pour la résolution spatiale visant à la meilleure définition de l'objet et pour les mesures de distance ou de surface. Le défaut de ce filtre est qu'il renforce certaines altérations de l'image et que le « bruit » de l'image ne permet pas un post-traitement 3D.

Technique de rendu où les densités choisies sont mises en évidence par des couleurs arbitraires : on attribue à chaque pixel une couleur en rapport à la mesure qu'il renferme - unité Hounsfield et une notion de transparence, ou pas, entres ces dernières.
- Filtre mou (passe-bas [B1os]) pour la résolution en densité afin de garder les valeurs d'atténuation des éléments constituant l'objet aux dépens des contours de l'objet qui deviennent « flous ». Ce filtre est parfait pour le post-traitement $3 \mathrm{D}$ des données (volumique, MIP et VR).
Grâce à une résolution extrêmement fine (200 microns), à l'utilisation des filtres de convolution et au rendu volumique ${ }^{\mathbf{1}}$, plusieurs types de reconstruction liés à des palettes de couleurs différentes permettent des rendus spécifiques (surfaciques ou volumiques) [ill. 3b et c]. Â la suite de l'acquisition et du choix de reformatage en frontal ou en sagittal, différents outils sont disponibles sur les images $2 \mathrm{D}$ : mesures de distance, d'angle, de surface et de densité. Après reconstruction $3 \mathrm{D}$, il également possible par « segmentation » de réaliser des découpes virtuelles au sein du modèle $3 \mathrm{D}$ pour isoler des éléments [ill. 3d], mais aussi de faire des calculs de volumes.

Dans certain cas, une altération du résultat peut être générée, qualifiée, en imagerie médicale, « d'artefact ». En général, ceci est dû à une absorption plus ou moins importante des rayons $\mathrm{X}$ par certains matériaux particulièrement denses (comme des éléments de nature métallique ou, dans le cadre de la céramique, par des éléments comme le quartz). La qualité de l'image dépend de l'appareil et de ses performances intrinsèques, mais aussi des choix de la personne qui réalise les images. En effet, si un grand nombre d'opérations complexes sont automatisées, il reste des choix techniques à effectuer concernant le champ et les épaisseurs de coupe, en fonction de la nature ou de la taille de l'objet et de la problématique de recherche.

\section{En pratique}

Dans la démarche de reconstitution des chaînes opératoires de fabrication de la poterie, l'identification et l'interprétation des traces visibles par observation directe jouent un rôle important. Depuis les premiers travaux de technologie comparée dans les années 1950, des études plus détaillées ou réalisées de manière systématique sur des corpus afin d'identifier des chaînes opératoires particulières se sont efforcées de mieux caractériser les macrotraces de façonnage ou de finition (Giligny, Méry, 2010). La nature des observations réalisées fait appel aux méthodes visuelles macroscopiques à faible grossissement, à l'observation de sections polies et de lames minces. La principale difficulté de ce type d'approche réside dans le fait que les traces de montage ne sont pas toujours très lisibles, notamment parce qu'elles ont été totalement ou partiellement oblitérées par les opérations ultérieures. Cet examen macroscopique caractérise un certain nombre de stigmates visibles à la surface des récipients (variation topographique, fissurations ou fracturations, textures) et des sections radiales des parois (aspect de la matrice argileuse, orientation préférentielle des éléments non plastiques, plan de fracturation). Dès les première études, le recours à l'imagerie aux rayons $\mathrm{X}$ pour le repérage des traces techniques de la fabrication des céramiques a été pratiqué afin 

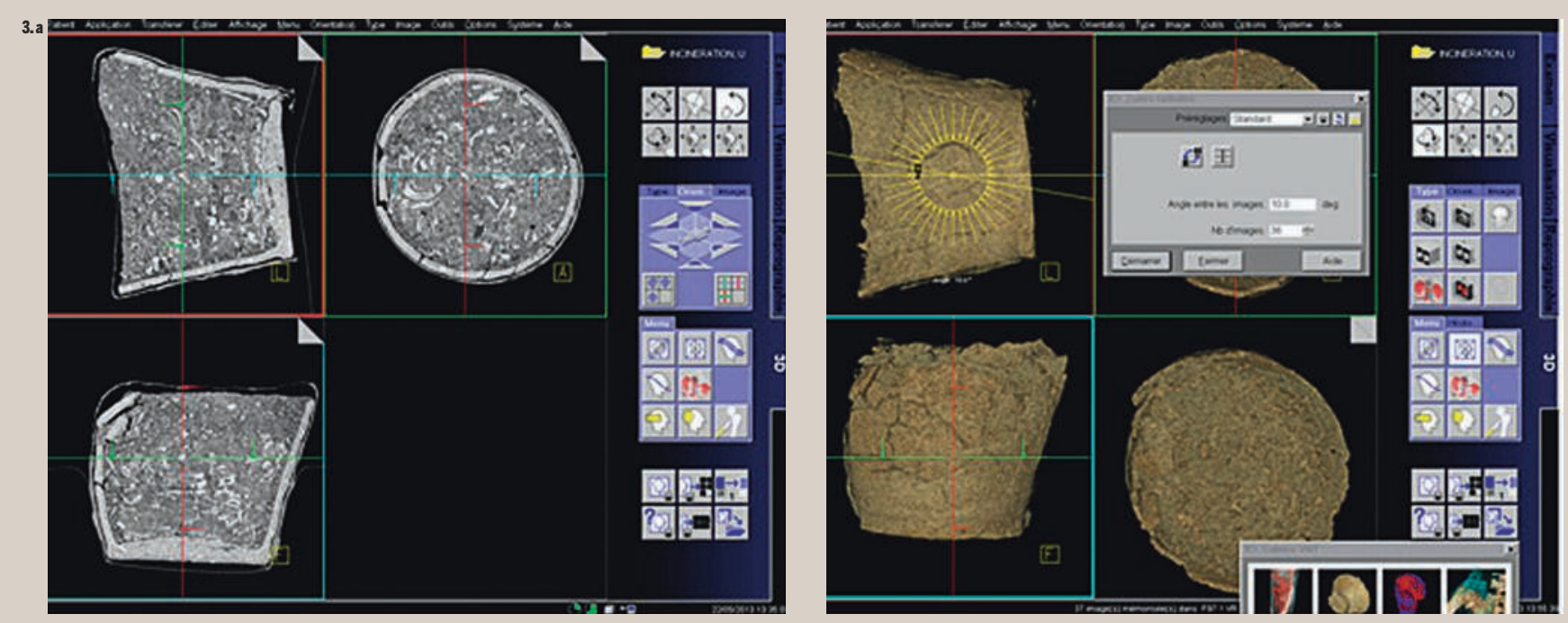

$\cong$
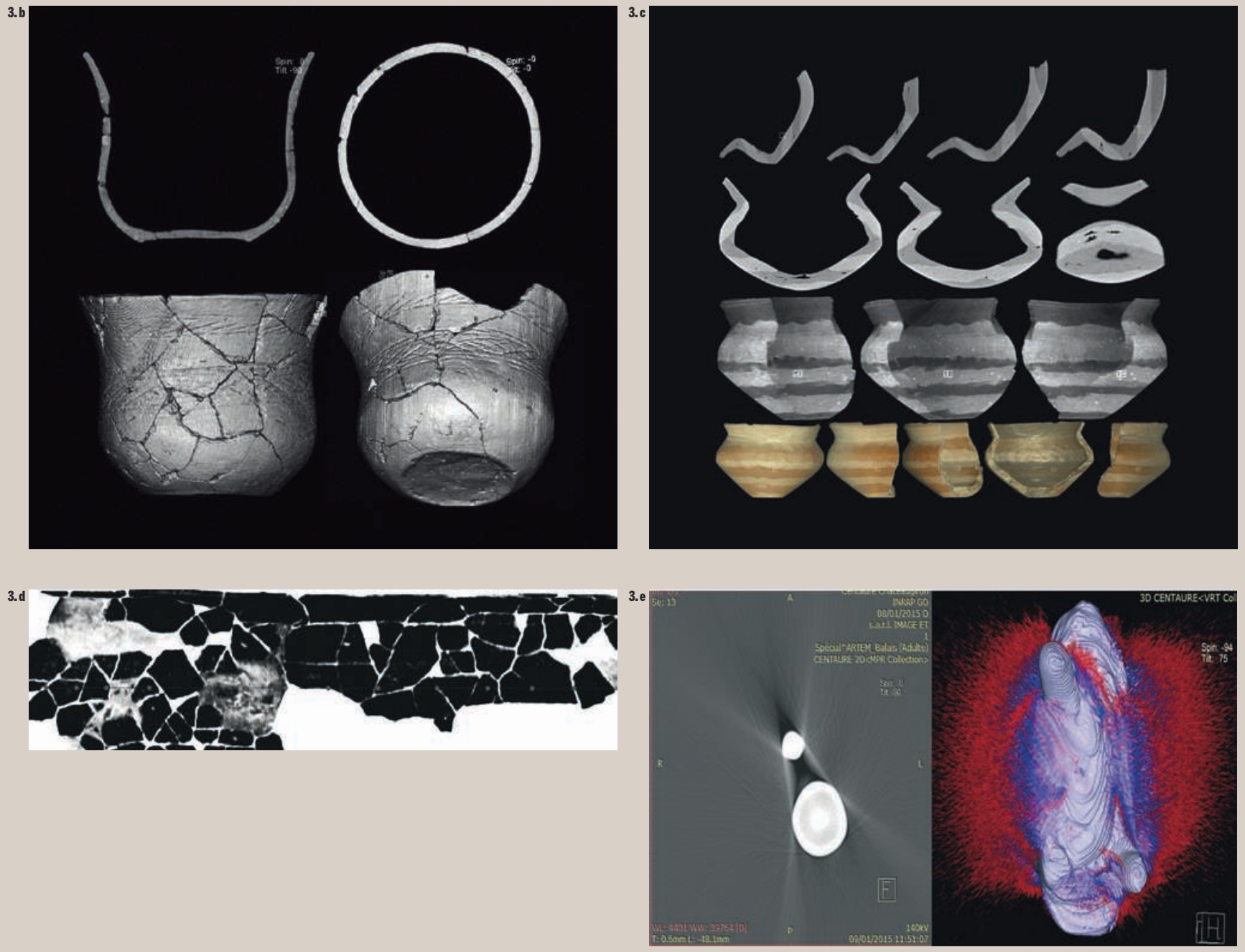
d'aller au-delà de l'analyse des éléments de surfaces (Rye, 1977 ; Van Doosselaere, 2005 ; Pierret, 1995). Cette technique d'imagerie de transmission par rayons X permet l'obtention d'un cliché dont le contraste dépend à la fois de l'épaisseur et du coefficient d'atténuation des éléments traversés. Cette technique ne peut fournir que des images projetées de l'ensemble du volume traversé par les rayons $\mathrm{X}$. La scanographie $\mathrm{X}$ résout donc le problème en réalisant pour la zone étudiée des images de coupes fines sous différents angles. En ce qui concerne la céramique, plusieurs travaux récents ont développé une analyse des techniques de façonnage ou des dégraissants grâce à la scanographie (Bouzakis et al., 2011 ; Kahl, Ramminger, 2012). Ces expérimentations ont montré la pertinence et l'efficacité de la tomodensitométrie pour mettre en évidence des structures à la surface et à l'intérieur de la pâte (identification et caractérisation - taille, densité, orientation - des éléments organiques et minéraux présents dans la matrice).

Après acquisition, il est possible de faire des analyses des images par coupes de o,5 $\mathrm{mm}$ sur les axes $x, y, z$, qui permettent de visualiser les traces de fabrication (épaisseur des parois, joints et raccords de colombins, étirements, mode de fabrication du fond), la fracturation et les fissures, les dégraissants, les décors et les inclusions [ill. 4a et b]. Des reconstructions $3 \mathrm{D}$, telles que le MIP (maximum-intensity projection), permettent de caractériser les matériaux qui composent la pâte : fragmentation, différences de densité, répartition, orientation, taille des éléments, porosité [ill. 4c]. Elles peuvent également modéliser les surfaces interne et externe des récipients. Le posttraitement des images optimise la visualisation des empreintes de doigts, voire d'outils, ce qui révèle les points de compressions ou d'étirements liés au façonnage, mais aussi permet de les corréler à des éléments de fracturation, à la répartition et/ou à l'orientation du dégraissant et à des porosités présentes au sein de la matrice argileuse [ill. $4 \mathrm{~d}$ et e]. La caractérisation des porosités se fait en restituant, par exemple, le volume de végétaux utilisés comme dégraissant à partir de leurs empreintes dans la pâte. Il est aussi possible de compléter les analyses visuelles en utilisant des logiciels de calcul, tels que Matlab, afin de réaliser des analyses qualitatives et quantitatives. On peut ainsi, par exemple, calculer des pourcentages (de densité et de porosité de la pâte ; de bulles d'air ou d'eau à l'intérieur de la pâte ; d'inclusions, de fissures et de dégraissants) et aussi faire des statistiques pour classer le dégraissant par grandeur, morphologie ou par densité. Par développement de l'objet, on peut également mesurer et classer, par section ou par ensemble, les différences de densité dans la distribution de la pâte. La méthode est non invasive, nécessite peu de manipulations et est très rapide de mise en œuvre, ce qui permet d'envisager de faire l'acquisition de corpus relativement conséquents (trois à quatre vases ou une centaine de tessons par heure) ; le post-traitement des images, lui, est contraint par la problématique de l'étude et le type d'échantillon.

Dans la démarche de reconstitution des chaînes opératoires de fabrication de la poterie, les différentes approches et échelles d'observation (examen macroscopique, microscopie optique, ou numérique-photogrammétrie, tomodensitométrie...) sont complémentaires ; seuls varient les conditions et les moyens mis en œuvre. Néanmoins, L'intérêt de la tomodensitométrie est de ne pas se cantonner à une analyse formelle, limitée à l'étude des formes, des décors et des stigmates technologiques visibles à la surface de la céramique. Il s'agit d'une méthode proposée comme traitement alternatif, où l'objet archéologique est remplacé par son modèle virtuel dans les étapes du processus après acquisition de données $3 \mathrm{D}$. L'objectif reste d'identifier et de restituer les modalités correspondant à l'intégralité des différentes étapes de la chaîne opératoire de production de la poterie, mais avec un plus grand degré de précision. Les données numériques $3 \mathrm{D}$ obtenues (réalité virtuelle, mixte ou augmentée) permettent en outre de concevoir des modalités de visualisation et d'interaction qui ouvrent des champs d'étude encore très peu explorés. On pourra, notamment et avec un autre regard, mettre en contexte l'objet étudié, que ce soit un contexte de fabrication, d'utilisation ou de découverte. 

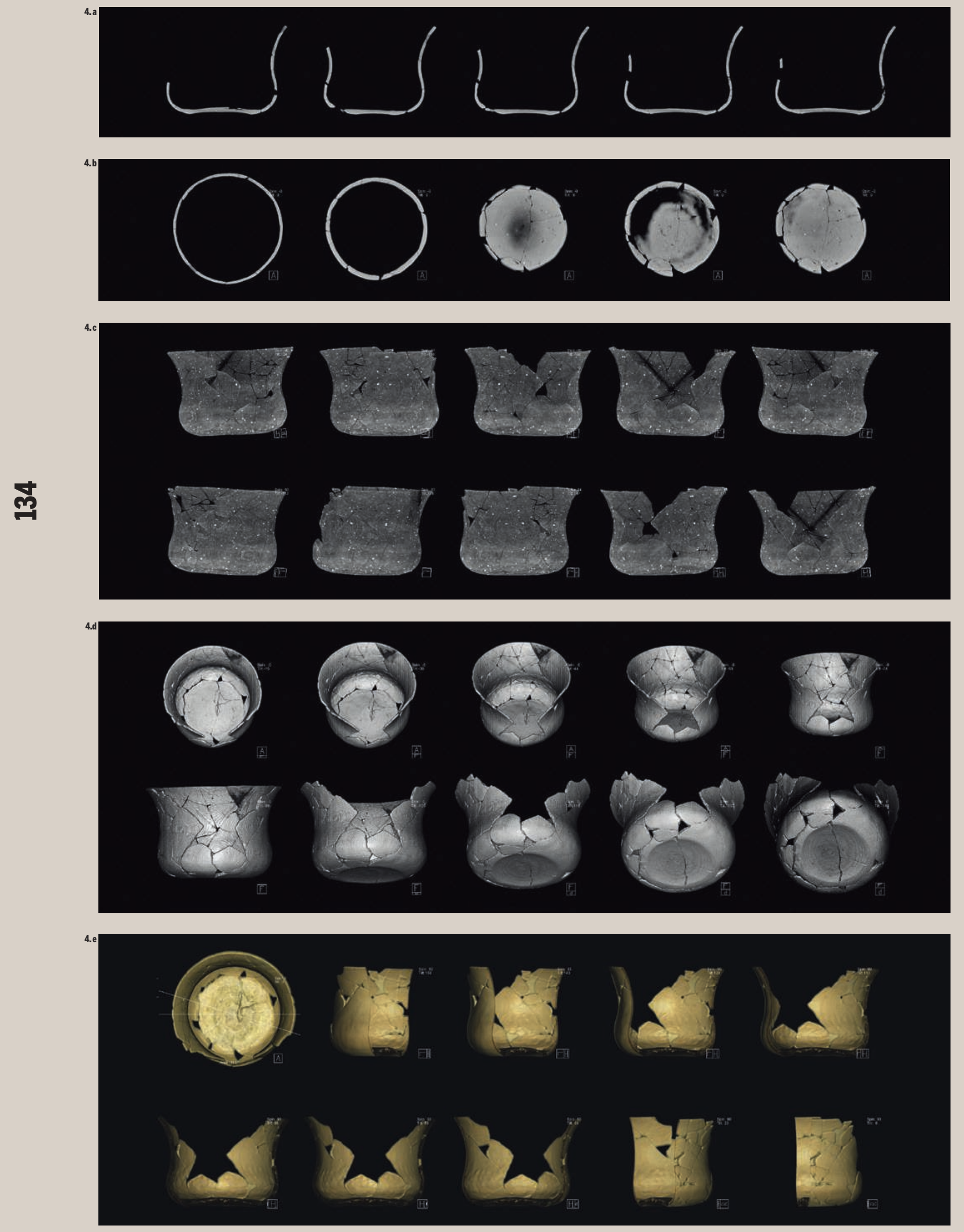


\section{Références bibliographiques}

Anderson T., Fell C., 1995, « Analysis of Roman cremation vessels by computerized tomography », Journal of Archaeological Science, vol. 22, 5 , p. 609-617.

Arbace L., Sonnino E., Callieri M., Dellepiane M. FABbri M., IDELSON A. I., SCOPIGNo R. 2013 « Innovative uses of ${ }_{3} \mathrm{D}$ digital technologies to assist the restoration of a fragmented terracotta statue ", Journal of Cultural Heritage, vol. 14, 4, p. 332-345.

Barreau J.-B., Nicolas T., Bruniaux G., Petit E. Petit Q., Bernard Y., Gaugne R., Gouranton V., 2014, « Photogrammetry Based Study of Ceramics Fragments », International Journal of Heritage in the Digital Era, 3 (4), p. 643-656.

Biron M., Hurtin S., Nicolas T., Tavernier C., 2015 « La tomographie des objets archéologiques complexes et/ou altérés : outil d'identification, d'analyse et d'aide à la décision pour les mesures conservatoires », in Restaurer l'ordinaire. Exposer l'extraordinaire? Journée des restaurateurs en archéologie (JRA), Arles, 2014, p. 41-44.

Boespflug X., Ross N., Long B. F., Dumais J. F., 1994,

« Tomodensitométrie axiale : relation entre l'intensité tomographique et la densité de la matière ", Revue canadienne des sciences de la Terre, t. 31 (2), p. 426-434.

Bouzakis K. D., Pantermalis D., Efstathiou K., Varitis E., Paradisiadis G., Mavroudis I., 2011, "An Investigation of Ceramic Forming Method Using Reverse Engineering Techniques: The Case of Oinochoai from Dion, Macedonia, Greece », Journal of Archaeological Method \& Theory, vol. 18, 2, p. 111-124.

Forte M., Kurrillo G., 2010, « Cyber-archaeology and met averse collaborative systems ", Metaverse Creativity, 1, 1, p. 7-19.

Giligny F., MÉry S., 2010, Approches de la chaîne opératoire de la céramique, Les Nouvelles de l'archéologie, 119. http://journals.openedition.org/ nda/950

HARVIG L., LyNNERUP N., AMSGa ARD EBSEN J, 2012, " Computed tomography and computed radiography of late Bronze Age cremation urns from Denmark: an interdisciplinary attempt to develop methods applied in bioarchaeological cremation research ", Archaeometry, 54 (2), p. 369-387.

HARVig L., LyNNERUP N., 2013, « On the volume of cremated remains: a comparative study of cremated bone volume measured manually and assessed by Computed Tomography », Journal of Archaeological Science, 40, p. 2713-2722.

Hassmann H., Heintgeis T., Rasink B., Winghart S WULF F.-W., 2012, « Der bronzezeitliche Hortfund von Gessel, Stadt Syke, Landkreis Diepholz, Denkmalplege », Berichte zur Denkmalpflege in Niedersachsen, 1, p. 23-28.
Houle-Wierzbicki Z., Treyvaud G., Raguin E., AUger R., Riвot I., 2015, « Development of the X-ray CT data base for the paleopathological analysis: Example of the St. Matthew protestant churchyard, Quebec City (1771-1861)», in $2^{\text {nd }}$ International Conference on Tomography of Materials and Structures, Québec, 29 juin2 juillet 2015

Hu S., Raza A., Min K., Kohn B. P., Reiners P. W., Ketcham R. A., WANG J., Gleadow A. J. W., 2006, « Late Mesozoic and Cenozoic Thermotectonic Evolution along a Transect from the North China Craton through the Quinling Orogen into the Yangtze Craton, Central China », Tectonics, 25, DOI 10.1029/2006TC001985

Huisman D. J., Ngan-Tillard D., Tensen M. A., RaEmaekers D., LaARMAN F. J., 2014, « A question of scales: Studying Neolithic subsistence using micro CT scanning of midden deposits ", Journal of Archaeological Science, 49, p. 585-594.

IKRAM S., Slabbert R, CORNelius I., DU Plessis A. Swanepoel L. C., Weber H., 2015, « Fatal forcefeeding or Gluttonous Gagging? The death of Kestrel SACHM 2575 ", Journal of Archaeological Science, 63, p. 72-77.

KAHL W.-A., RAMminger B., 2012, « Non-destructive fabric analysis of prehistoric pottery using highresolution X-ray microtomography: a pilot study on the late Mesolithic to Neolithic site HamburgBoberg », Journal of Archaeological Science, 39, p. 2206-2219.

KaK A.C., Slaney M., 1999, Principles of Computerized Tomographic Imaging, IEEE Press, New York.

Ketcham R. A., Carlson W. D., 2001, « Acquisition, Optimization and Interpretation of X-ray Computed Tomographic Imagery: Applications to the Geosciences », Computers and Geosciences, 27, p. 381-400.

Ketcham R. A., ItURRINO G. J., 2005, « Nondestructive High-resolution Visualization and Measurement of Anisotropic Effective Porosity in Complex Lithologies Using High-resolution X-ray Computed Tomography », Journal of Hydrology, 302, p. 92-106.

Lee S. S., Gantzer C. J., Thompson A. L., Anderson S. H., Кетснам R. A., 2008, « Soil Surface-sea Characterization Using Analysis of High-resolution Computed Tomography », Soil Science Society of America Journal, 72, 5, p. 1478-1485.

Le Puil-Texier M., Nicolas T, TaVernier C, 2015 « L'apport de l'examen tomodensitométrique à la fouille et l'analyse des dépôts de crémation en urne », in Nouvelles approches de l'archéologie funéraire, $6^{e}$ rencontre du Gaaf, Paris, 2014.

Minozzi S., Giuffra V., Bagnoli J., Paribeni E., Giustini D., Caramella D., Fornaciari G., 2010, "An investigation of Etruscan cremations by Computed Tomography (CT) ", Antiquity, 84, p. 195-201.

Mödlinger M., 2008, « Micro-X-ray Computer Tomography in Archaeology: Analyses of a Bronze Age Sword », Insight - Non-Destructive Testing and Condition Monitoring, 5o/5, p. 323-326.
Nicolas T., Gaugne R., Tavernier C., Gouranton V., ARnaldi B., 2014, « Preservative approach to study encased archaeological artefacts », in International Conference on Cultural Heritage, Euromed, Limassol, Cyprus, November 2014, Lecture Notes in Computer Science, 8740, p. 332-341.

Nicolas T., Gaugne R., Tavernier C., Petit Q., Gouranton V., Arnaldi B., 2015, « Touching and interacting with inaccessible Cultural Heritage ", Presence, Teleoperators and Virtual Environments, MIT Press, vol. 24, 3.

Pierret A., 1995, Analyse technologique des céramiques archéologiques : développements méthodologiques pour l'identification des techniques de façonnage. Un exemple d'application, le matériel du village des Arènes à Levroux (Indre), Thèse l'université de Paris I, Panthéon-Sorbonne.

Re A., Corsi J., Demmelbauer M., Martini M., Mila G., RiCCI C., 2015, « X-ray tomography of a soil block: a useful tool for the restoration of archaeological finds ", Heritage Science, 3:4, Science Letters, 246, p. 102-108.

Rye O. S., 1977, « Pottery manufacturing techniques: X-ray Studies », Archaeometry, vol. 19, 2, p. 205-211.

Stelzner J., Ebinger-Rist N., Peek C., Schillinger B., 2010, « The application of 3 D computed tomography with X-rays and neutrons to visualize archaeological objects in blocks of soil », Studies in Conservation, vol. 55, n 2, p. 95-106.

Treyvaud G., 2010, « Doubles témoins de l'histoire, étude technologique en archéométallurgie », Archéologiques, 23, Revue de l'Association des Archéologues du Québec.

Treyvaud G., 2015, « The use of metals and metal products on urban and rural archaeological sites: reconstructing technologies employed by native american and european artisans in New France ", in International Conference on Tomography of Materials and Structures, Québec, Canada.

Treyvaud G., Dupras S., Auger R., 2013, « De l'Égypte à Québec, un quatuor d'oudja à l'îlot des Palais ", Archéologiques, 26, Revue de l'Association des Archéologues du Québec.

XIUjIE W., LyNNE A., 2009, « Schepartz, Application of computed tomography in paleoanthropological research », Progress in Natural Science, vol. 19, $n^{\circ} 8$, p. 913-921.

VAn Doosselaere B., 2005, « Stylistic perception and ceramic technology: shaping processes of archaeological pottery from Koumbi Saleh (Mauritanie, IXth-XVth centuries), reconstitution and interpretation ", in Proceedings of the XVIIth UISPP Congress, Cambridge Monographs in Archaeology, BAR International Series, Oxford.

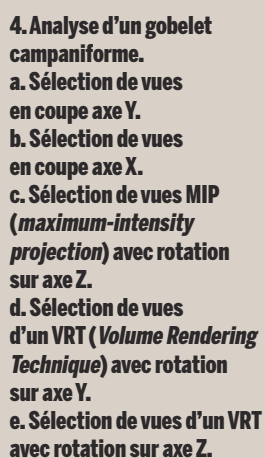

